\title{
Interaction between survivin and aurora-B kinase plays an important role in survivin-mediated up-regulation of human telomerase reverse transcriptase expression
}

\author{
MOMOKO FURUYA*, NAOKI TSUJI*, DAISUKE KOBAYASHI and NAOKI WATANABE \\ Department of Clinical Laboratory Medicine, Sapporo Medical University \\ School of Medicine, South-1, West-16, Chuo-ku, Sapporo 060-8543, Japan
}

Received September 10, 2008; Accepted November 25, 2008

DOI: 10.3892/ijo_00000232

\begin{abstract}
Survivin, a member of the apoptosis inhibitor family, shows increased expression in human cancers of various origins. It has been demonstrated that survivin inhibits apoptosis via caspase inhibition and promotes mitosis via aurora-B kinase activation. We recently reported that survivin enhances the expression of human telomerase reverse transcriptase (hTERT), a major determinant of telomerase activity in colon cancer cells. Survivin up-regulates hTERT expression by promoting the expression of specificity protein-1 (Sp1)- and c-Myc-mediated gene transcription via enhancing the phosphorylation of these transcriptional factors. However, the mechanism by which survivin regulates the phosphorylation of Sp1 and c-Myc is not well defined. In the present study, we hypothesized that survivin promotes the phosphorylation of $\mathrm{Sp} 1$ and c-Myc by activating aurora-B kinase. Inhibition of this enzyme by introducing small inhibitory RNA attenuated the phosphorylation of Sp1 and c-Myc and resulted in the abolition of the survivin effect on hTERT expression. In addition, blocking survivin phosphorylation at a threonine residue by inhibiting cyclin-dependent kinase 1 caused the dissociation of aurora-B kinase from survivin and attenuated the up-regulation of hTERT expression by survivin. Taken together, these results suggest that the interaction between
\end{abstract}

Correspondence to: Dr Naoki Watanabe, Department of Clinical Laboratory Medicine, Sapporo Medical University School of Medicine, South-1, West-16, Chuo-ku, Sapporo 060-8543, Japan E-mail: watanabn@sapmed.ac.jp

*Contributed equally

Abbreviations: FasL, Fas ligand; hTERT, human telomerase reverse transcriptase; Sp1, specificity protein 1; CDK1, cyclin-dependent kinase 1; FBS, fetal bovine serum; siRNA, small inhibitory RNA; RT-PCR, reverse transcription polymerase chain reaction; rRNA, ribosomal RNA; EMSA, electrophoretic mobility shift assay; PBS, phosphate-buffered saline

Key words: survivin, aurora-B kinase, telomerase survivin and aurora-B kinase may be essential for survivin to increase hTERT expression.

\section{Introduction}

Survivin, a member of the apoptosis inhibitor family, is expressed in the embryonic and fetal organs but has not been identified in differentiated normal tissues, except in those of the thymus, basal colonic epithelium, endothelial cells and neural stem cells. Elevated levels of survivin expression were observed in human cancers of various origins such as the breast $(1)$, esophagus $(2)$, stomach $(3,4)$, colon $(5,6)$, pancreas (7), liver (8), uterus (9) and ovaries (10) as well as in leukemias (11).

It has been demonstrated that survivin inhibits the activation of downstream effectors of apoptosis, namely, caspase- 3 and caspase-7, in cells exposed to apoptotic stimuli (12-15). Since survivin overexpression desensitized cancer cells to several anticancer agents (12) and irradiation (15) that induce apoptosis, it acts as a resistance factor against these cancer treatment modalities.

Li et al demonstrated that survivin is expressed in the $\mathrm{G} 2 / \mathrm{M}$ phase of the cell cycle in a cycle-regulated manner (16). Bolton et al and Chen et al showed that survivin interacts with aurora-B kinase, a serine/threonine kinase involved in the regulation of mitosis and enhances its activity $(17,18)$. These results suggest that survivin promotes mitosis via aurora-B kinase activation and contributes to the aberrant growth of cancer cells.

We recently reported that survivin enhances Fas ligand (FasL) expression in colon cancer cells. This indicates that survivin enables cancer cells to counterattack immune cells by inducing FasL-triggered apoptosis in the cells of the immune surveillance system (19). In addition, we observed that survivin up-regulates human telomerase reverse transcriptase (hTERT), a major determinant of telomerase activity and maintains the telomere length, indicating that survivin prolongs the cellular lifespan in cancer (20). These findings portray survivin as a multifunctional protein that is important for the proliferation of cancer cells in vivo.

Survivin up-regulates hTERT expression by promoting specificity protein-1 (Sp1)- and c-Myc-mediated gene transcription via enhancing their DNA binding to the hTERT gene promoter. Moreover, survivin increases the 
phosphorylation of these transcriptional factors at serine and threonine residues, thereby up-regulating their DNA-binding activity (20). However, the precise mechanism by which survivin regulates the phosphorylation of $\mathrm{Sp} 1$ and c-Myc is not well defined.

In the present study, we hypothesized that survivin promotes the phosphorylation of $\mathrm{Sp} 1$ and $\mathrm{c}-\mathrm{Myc}$ by the activation of aurora-B kinase. Inhibition of this enzyme attenuated the phosphorylation of Sp1 and c-Myc and resulted in the abolition of the effect of survivin on hTERT expression. We also observed that blocking survivin phosphorylation at the threonine residue by inhibiting $\mathrm{p} 34^{\mathrm{cdc} 2}$ (cyclin-dependent kinase 1; CDK1) caused the dissociation of aurora-B kinase from survivin and attenuated the up-regulation of hTERT expression by survivin. Taken together, these results suggest that the interaction between survivin and aurora-B kinase may be essential for survivin to increase hTERT expression.

\section{Materials and methods}

Materials. Anti-hTERT antibody was from Calbiochem (San Diego, CA). Anti-survivin antibody for immunoblot analysis was from R\&D Systems (McKinley Place, NE). Anti-survivin antibody for immunoprecipitation, anti-CDK1 antibody, anti-Sp1 antibody, anti-c-myc antibody and antiactin antibody were from Santa Cruz Biotechnology (Santa Cruz, CA). Anti-aurora-B kinase antibody was from BectonDickinson (Franklin Lakes, NJ). Anti-phosphoserine antibody and anti-phospho-threonine antibody were from Chemicon International (Temecula, CA).

Cell culture. The human colon cancer cell line, LS180, was obtained from the American Type Culture Collection (Manassas, VA). This cell line was cultured in Dulbecco's modified Eagle's medium (DMEM; Sigma-Aldrich, St. Louis, MO) supplemented with $10 \%$ heat-inactivated fetal bovine serum (FBS; Invitrogen, Carlsbad, CA) and grown at $37^{\circ} \mathrm{C}$ in a humidified atmosphere of $5 \% \mathrm{CO}_{2}$.

Transfection of survivin gene into colon cancer cells. The plasmid encoding human survivin pcDNA3-myc-survivin and the vector control pcDNA3-myc were kindly provided by Dr John C. Reed (The Burnham Institute, La Jolla, CA). Transfections were performed using the Lipofectamine Plus reagent (Invitrogen) according to the manufacturer's protocol. Geneticin-resistant clones were used in this study.

Immunoblot analysis. Cytoplasmic and nuclear proteins were extracted from cells using NE-PER nuclear and cytoplasmic extraction reagents (Pierce, Rockford, IL) with a protease inhibitor cocktail (Sigma-Aldrich). Equal amounts of proteins were suspended in Tris-glycine SDS sample buffer (Invitrogen) with $0.05 \mathrm{M}$ dithiothreitol and were separated on a 4 to $20 \%$ gradient Tris-glycine gel (Invitrogen) under denaturing conditions using Tris-glycine SDS running buffer (Invitrogen). For detection of hTERT protein, 6\% Tris-glycine gel (Invitrogen) was used. Proteins were electroblotted to a nitrocellulose membrane (Invitrogen). Proteins were detected using a WesternBreeze chemiluminescent immunodetection kit (Invitrogen) according to the manufacturer's protocol.
Chemiluminescent was detected by radiographic film or ChemiDoc XRS (Bio-Rad Laboratories, Hercules, CA).

Transfection of siRNA for aurora-B kinase and CDK1 into colon cancer cells. A small inhibitory RNA (siRNA) were designed to target the coding region of the aurora-B kinase gene (nucleotides 598 to 618 , relative to the start codon) and CDK1 gene (nucleotides 123 to 143 , relative to the start codon), respectively. As the transfection control, we used non-silencing siRNA which has no homology to any known mammalian gene. The siRNA duplexes used in this study were as follows. For aurora-B kinase, 5'-GUG GGA CAC CCG ACA UCU UAA-3' and 5'-AAG AUG UCG GGU GUC CCA CUG-3'. For CDK1, 5'-GGA AGG GGU UCC UAG UAC UGC-3' and 5'-AGU ACUA GGA ACC CCU UCC UC-3'. Transfection of these siRNAs was performed using the HiPerFect transfection reagnet (Qiagen, Valencia, CA) according to the manufacturer's protocol.

Quantification of survivin and hTERT $m R N A$. The expression of survivin and hTERT mRNA was determined by quantitative reverse transcription polymerase chain reaction (RT-PCR) using an ABI PRISM 7700 Sequence Detection System (Applied Biosystems, Foster City, CA), as described previously $(19,20)$. The gene-specific primers and fluorescent hybridization probes for these genes used in quantitative RT-PCR were as follows. For survivin, the forward primer was 5'-AAG AAC TGG CCC TTC TTG GA-3'; reverse primer, 5'-CAA CCG GAC GAA TGC TTT T-3'; and probe, 5'-(FAM) CCA GAT GAC GAC CCC ATA GAG GAA CA (TAMRA)-3'. For hTERT, the forward primer was 5'-GGG CGC GTA CGA CAC CAT-3'; reverse primer, 5'-ATG TAC GGC TGG AGG TCT GTC A-3'; and probe, 5'-(FAM) CGG AGG TCA TCG CCA GCA TCA TC (TAMRA)-3'. Two splice variants of surviving, survivin-2B (retaining a part of intron 2 as a cryptic exon) and survivin- $\Delta \mathrm{Ex} 3$ (lacking exon 3 ), were not detected by this set of primers and probe (21). The amounts of these mRNAs were normalized as ratios to the amounts of 18S ribosomal RNA (rRNA), which was quantified using a Taq Man ribosomal RNA control reagents (Applied Biosystems). PCR products were also confirmed by agarose gel electrophoresis.

Electrophoretic mobility shift assay (EMSA). Nuclear proteins were extracted from the cells using NE-PER nuclear and cytoplasmic extraction reagents (Pierce) along with a protease inhibitor cocktail (Sigma-Aldrich). Oligonucleotide probes containing the Sp1-binding motif of the hTERT gene promoter (5'-TCC TTT CCG CGG CCC CGC CCT CTC CTC GCG GCG CGA-3') and c-Myc-binding motif of the hTERT gene promoter (5'-GCG CTT CCC ACG TGG CGG AGG G-3') were prepared; these were 3 ' end-labeled with biotin from Sigma Genosys Japan (Ishikari, Japan). Detection of Sp1- and c-Myc-oligonucleotide complexes was performed using the LightShift Chemiluminescent EMSA kit (Pierce) according to the manufacturer's protocol. The specificity of Sp1 or c-My DNA binding was determined by competition reactions in which a 200-fold molar excess of unlabeled oligonucleotide was added to the binding reaction. Products of the binding reactions were resolved by electrophoresis on a $6 \%$ 
polyacrylamide gel (Invitrogen) using 1/2X TBE buffer (Invitrogen). The protein-oligonucleotide complexes obtained were electroblotted to a nylon membrane (Invitrogen). After incubation in blocking buffer at room temperature for $15 \mathrm{~min}$, the membrane was incubated with a streptavidin-horseradish peroxidase conjugate at room temperature for $15 \mathrm{~min}$. The membrane was incubated with a chemiluminescent substrate for $5 \mathrm{~min}$.

Immunoprecipitation. Nuclear proteins (350-500 $\mu \mathrm{g}$ ) extracted from the cells were immuno-precipitated by incubating with $20 \mu 1$ of Protein G PLUS-Agarose (Santa Cruz Biotechnology) and $4 \mu \mathrm{g}$ of rabbit anti-Sp1 antibody, anti-c-Myc antibody, or anti-survivin antibody in phosphate-buffered saline (PBS) overnight at $4^{\circ} \mathrm{C}$. The protein-antibody-protein $\mathrm{G}$ complexes were pelleted at $1,000 \mathrm{x} \mathrm{g}$ and then washed four times with PBS. Immunoprecipitates were resuspended in the Trisglycine SDS sample buffer (Invitrogen) with $0.05 \mathrm{M}$ dithiothreitol and boiled for $3 \mathrm{~min}$. The immunoprecipitate was then electrophoresed on a 4-20\% gradient Tris-glycine gel (Invitrogen) and immunoblot analysis was performed.

\section{Results}

Survivin up-regulates hTERT expression. To confirm that survivin up-regulates hTERT expression, we introduced the survivin gene expression vector pcDNA3-myc-survivin into LS180 cells and examined its effect on hTERT protein expression by using immunoblot analysis. Since the survivin protein is localized in both the cytosol and nucleus, we determined its expression in both these sites. As shown in Fig. 1A, the two survivin gene transfectants F1 and F15 confirmed the presence of both the myc-tagged survivin protein and the endogenous survivin protein in the cytosol and nucleus. Up-regulation of hTERT protein expression was higher in these survivin gene transfectants as compared to the control vector transfectants (vector control) and extent of hTERT protein up-regulation appeared to depend on the level of myc-tagged survivin protein expression. Up-regulation of hTERT mRNA was also observed in survivin gene transfectants (Fig. 1B).

Survivin-mediated hTERT up-regulation was attenuated by the inhibition of aurora-B kinase expression. It has been reported that survivin interacts with the mitotic regulator aurora-B kinase and enhances its activity $(18,19)$. To clarify whether aurora-B kinase is required for survivin to enhance hTERT expression, we introduced siRNA targeting the aurora-B kinase gene (ABK-siRNA) into the survivin gene transfectant F1 and determined hTERT protein expression by using immunoblot analysis. As illustrated in Fig. 2A, the introduction of ABK-siRNA inhibited aurora-B kinase protein expression but not endogenous and myc-tagged survivin protein expression. The hTERT protein expression was greater in survivin gene transfectants than in control vector transfectants after introducing the non-silencing siRNA. However, when ABK-siRNA was introduced into the survivin gene transfectant, enhanced hTERT protein expression was attenuated. A similar pattern was noted in hTERT mRNA expression (Fig. 2B). These results indicate that the up-
A

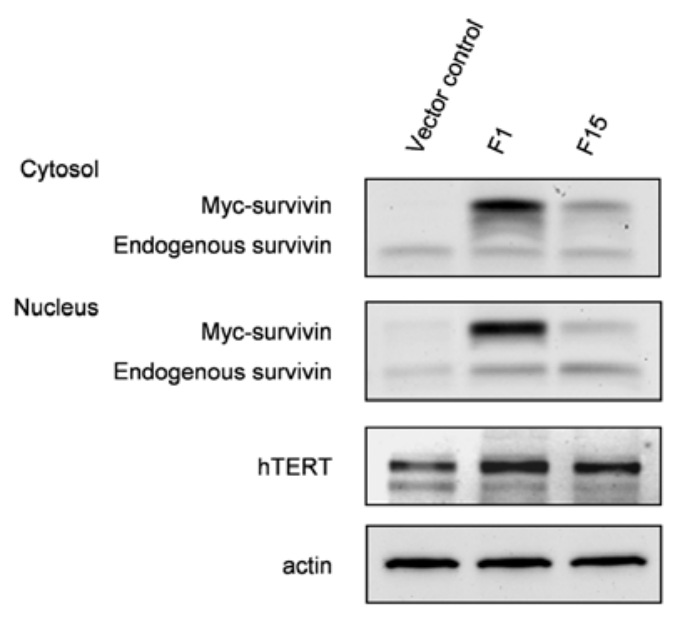

B

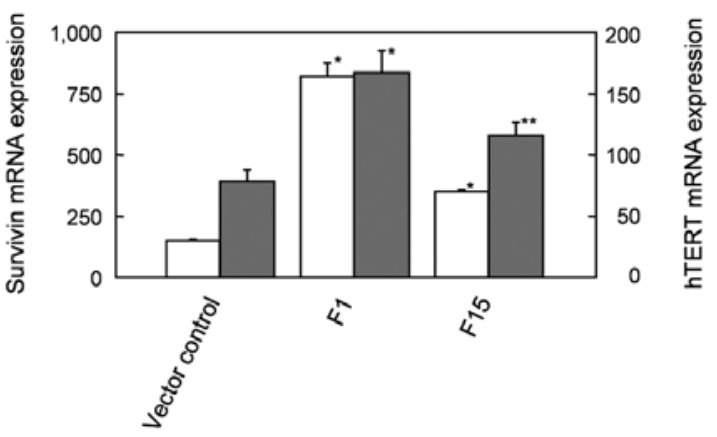

Figure 1. Survivin up-regulated hTERT expression. LS180 cells are transfected with pcDNA3-myc-survivin or pcDNA3-myc. Expression of survivin and hTERT proteins (A) or mRNA (B) in control vector transfectants (vector control) and survivin gene transfectants (F1 and F15) were examined using immunoblot analysis and quantitative RT-PCR, respectively. The amounts of mRNA were normalized as ratios to the amount of $18 \mathrm{~S}$ rRNA. Data are represented as mean $\pm \mathrm{SD}$. Open bars, survivin mRNA; gray bars, hTERT mRNA. ${ }^{*} \mathrm{P}<0.001,{ }^{* *} \mathrm{P}<0.01$, as evaluated using t-test.

regulation of hTERT expression by survivin requires the aurora-B kinase.

Inhibition of aurora-B kinase expression abolished the enhancement of Spl-and c-Myc-mediated hTERT gene transcription by survivin. Since the inhibition of aurora-B kinase abolished the ability of survivin to up-regulate hTERT mRNA expression, aurora-B kinase may be involved in the transcriptional regulation of hTERT by survivin. It has been demonstrated that the transcription factors Sp1 and c-Myc were required for basal transcription from the hTERT gene promoter (22). We previously showed that the binding of Sp1 and c-Myc to the hTERT gene promoter was enhanced by survivin (20). In addition, survivin promoted the phosphorylation of these two transcriptional factors at serine and threonine residues, thereby increasing their DNA-binding activity (23-25). Therefore, we investigated the DNA binding of Sp1 and c-Myc to the hTERT gene promoter in the ABKsiRNA transfectants by using EMSA. In this assay, we used two types of biotinated oligonucleotides, one containing the 
A

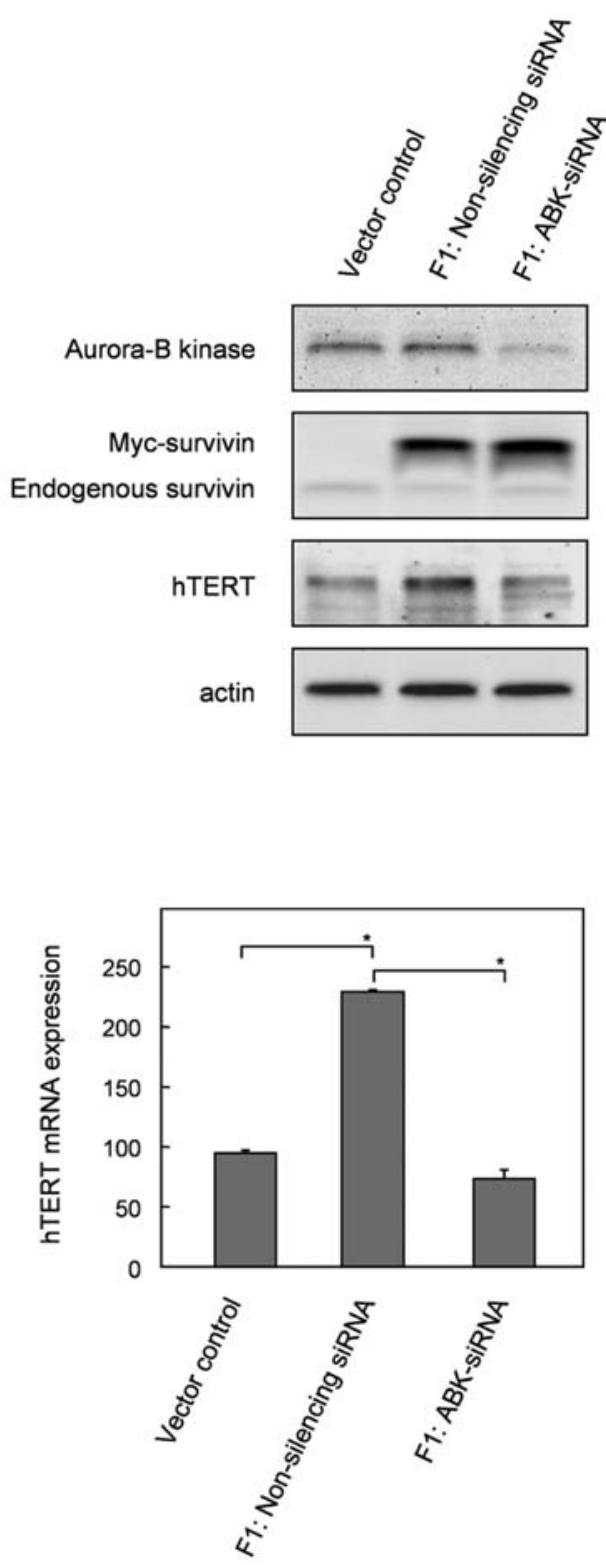

Figure 2. Survivin-mediated hTERT up-regulation was attenuated by the inhibition of aurora-B kinase expression. The survivin gene transfectant F1 was transfected with non-silencing siRNA, which does not share its homology with any known mammalian gene, or siRNA targeting the aurora-B kinase gene (ABK-siRNA). Expression of indicated proteins (A) and hTERT mRNA (B) were assessed at 6 days after transfection by immunoblot analysis or quantitative RT-PCR, respectively. The amounts of mRNA were normalized as ratios to the amount of $18 \mathrm{~S}$ rRNA. Data are represented as mean \pm SD. Vector control, control vector transfectants. ${ }^{*} \mathrm{P}<0.001$, as evaluated using t-test.

Sp1-binding motif and the other, the c-Myc-binding motif. Each oligonucleotide spanned the nucleotide positions from -21 to +14 , or -173 to -152 relative to the hTERT translation initiation site. As shown in Fig. 3A and B, survivin gene transfectants showed higher DNA binding of Sp1 and c-Myc as compared to the control vector transfectants even after the introduction of non-silencing siRNA. On the other hand, introduction of ABK-siRNA resulted in decreased DNA binding of these two transcriptional factors in survivin gene transfectants.
A

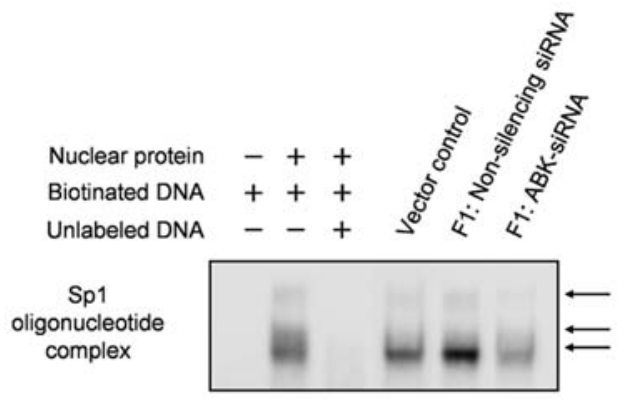

B

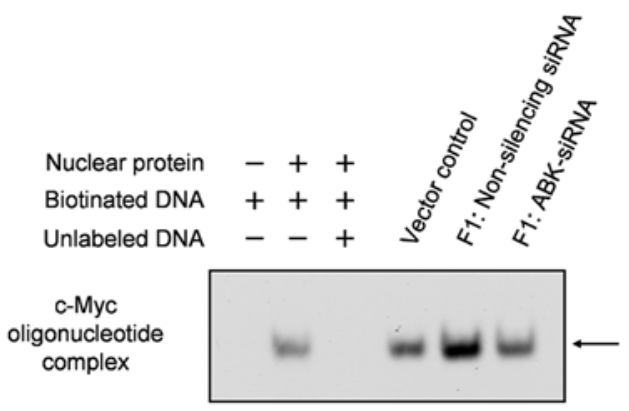

Figure 3. Enhanced DNA binding of Sp1 and c-Myc to the hTERT gene promoter in survivin gene transfectants was reduced by the inhibition of aurora-B kinase. The survivin gene transfectant F1 was transfected with non-silencing siRNA, which does not share its homology with any known mammalian gene, or siRNA targeting the aurora-B kinase gene (ABKsiRNA). DNA binding of Sp1 (A) and c-Myc (B) to the hTERT gene promoter was examined at 6 days after transfection by EMSA. Arrows indicate the protein-oligonucleotide complexes. Vector control, control vector transfectants.

Next, we determined the amounts of the phosphorylated Sp1 and c-Myc protein in the ABK-siRNA transfectants. Sp1 and $\mathrm{c}-\mathrm{Myc}$ proteins were immunoprecipitated from nuclear extracts of the transfectants by using the anti-Sp1 or c-Myc antibody. The samples were adjusted such that they contained an equal amount of $\mathrm{Sp} 1$ or c-Myc protein; subsequently, phosphorylated Sp1 and c-Myc protein expression was analyzed by immunoblot analysis using the anti-phosphoserine or anti-phosphothreonine antibody and the anti-phosphorylated c-Myc antibody, respectively. As shown in Fig. 4A and B, the expression of phosphorylated $\mathrm{Sp} 1$ and $\mathrm{c}-\mathrm{Myc}$ protein in survivin gene transfectants transfected with non-silencing siRNA was higher than that in the control vector transfectant. When ABK-siRNA was introduced into the survivin gene transfectants, the enhanced phosphorylated expression of these two proteins was clearly decreased. These results suggest that aurora-B kinase plays an important role in the promotion of Sp1 and c-Myc-mediated hTERT gene transcription by survivin.

Inhibition of survivin phosphorylation resulted in the loss of survivin effect on the up-regulation of hTERT expression. O'Connor et al demonstrated that CDK1-cyclin B1 phosphorylates survivin at threonine 34 and this phosphorylation is required for the inhibition of apoptosis during cell division (26). To investigate the role of survivin phosphorylation in the up-regulation of hTERT expression, we introduced siRNA targeted to the CDK1 gene into survivin gene transfectants. 
A

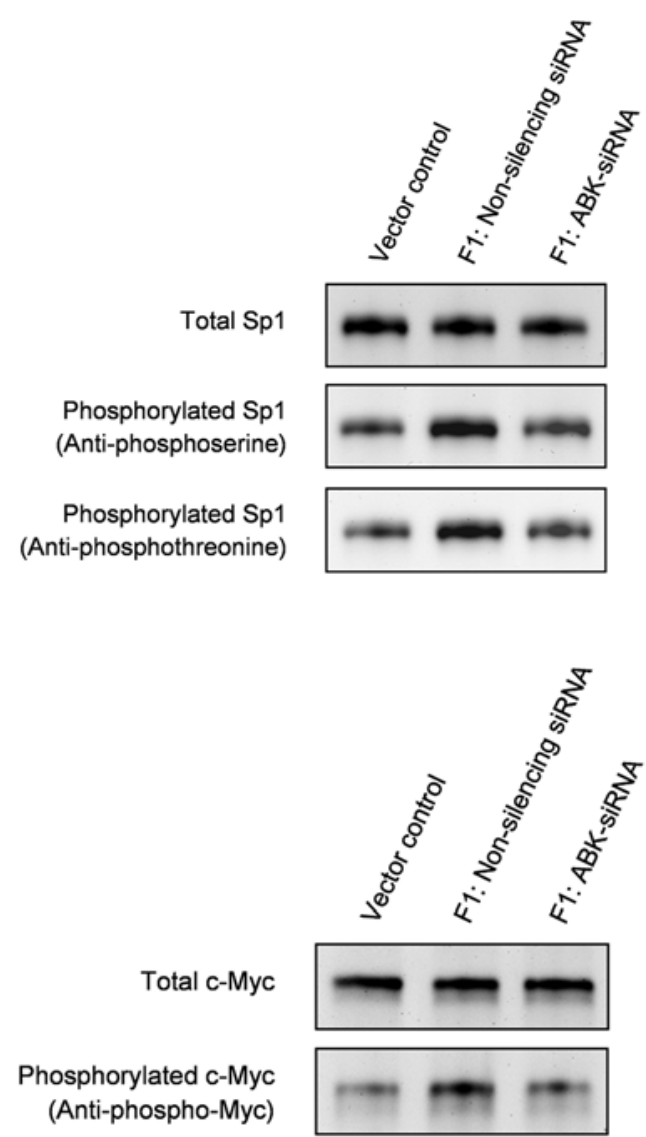

Figure 4. Survivin-mediated phosphorylation of Sp1 and c-Myc protein was attenuated by the inhibition of aurora-B kinase. The survivin gene transfectant F1 was transfected with non-silencing siRNA, which does not share its homology with any known mammalian gene, or siRNA targeting the aurora-B kinase gene (ABK-siRNA). Sp1 protein (A) and c-Myc protein (B) from nuclear extracts were immunoprecipitated at 6 days after transfection by using the anti-Sp1 antibody or anti-c-Myc antibody, respectively. Sp1 immunoprecipitates and c-Myc immunoprecipitates were immunoblotted using the anti-Sp1, anti-phosphoserine, or anti-phosphothreonine antibody and anti-c-Myc or anti-phosphorylated c-Myc antibody, respectively. Vector control, control vector transfectants.

As shown in Fig. 5A, although there was no difference in the level of CDK1 protein expression between the control vector transfectants and survivin gene transfectants transfected with non-silencing siRNA, the introduction of CDK1-siRNA reduced CDK1 protein expression. The levels of endogenous and myc-tagged survivin proteins were not affected by the introduction of CDK1-siRNA. In contrast, the enhanced expression of the hTERT protein in the survivin gene transfectant was decreased after the introduction of CDK1siRNA as compared to the non-silencing siRNA transfectant. A similar pattern was noted in hTERT mRNA expression (Fig. 5B).

To confirm that the introduction of CDK1-siRNA suppressed phosphorylation of the survivin protein, we determined the level of phosphorylated survivin protein expression in the CDK1-siRNA transfectant (Fig. 5C). Since the CDK1 protein is localized in the nucleus, survivin proteins from the nuclear extracts of transfectants were immunoprecipitated using an anti-survivin antibody. After adjustment of the samples to ensure that they contained an equal amount of survivin protein, phosphorylated survivin protein expression
$\mathbf{A}$

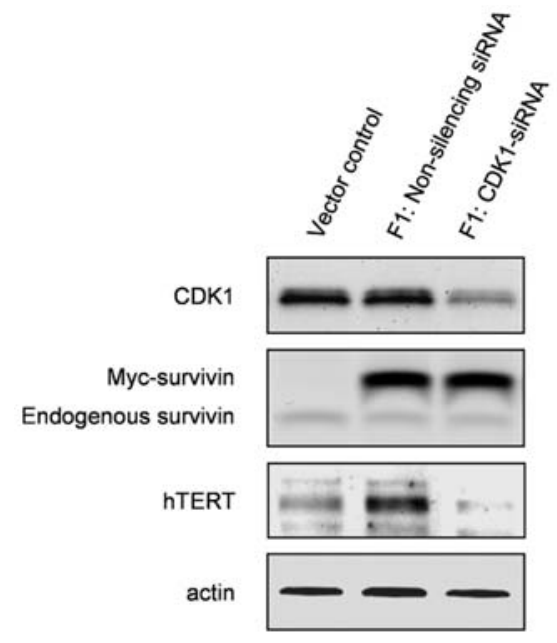

B

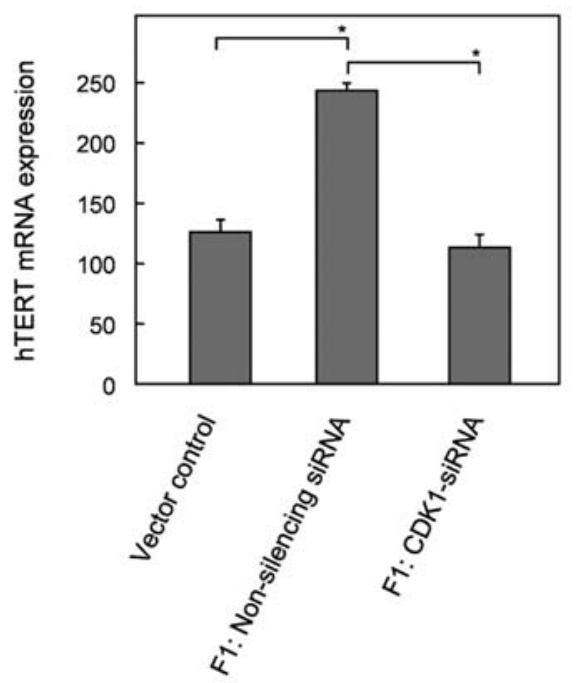

C

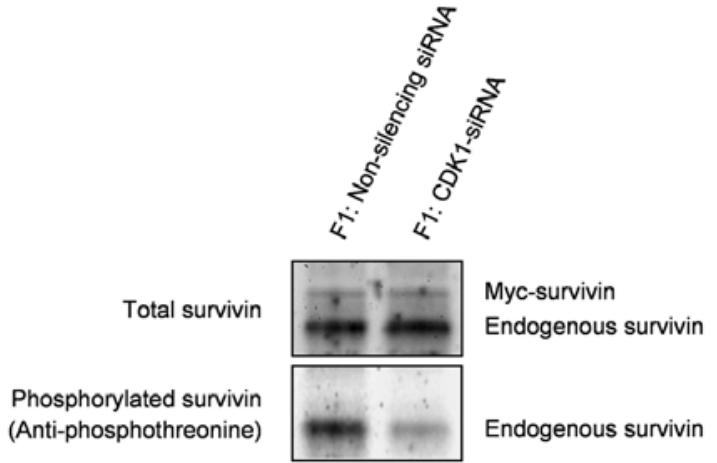

Figure 5. Survivin-mediated hTERT up-regulation was attenuated by the inhibition of survivin phosphorylation. Effect of CDK1 inhibition on the hTERT expression in survivin gene transfectants (A and B). The survivin gene transfectant F1 was transfected with non-silencing siRNA, which does not share its homology with any known mammalian gene, or siRNA targeting the CDK1 gene (CDK1-siRNA). Expression of indicated proteins (A) and hTERT mRNA (B) were assessed at 6 days after transfection by immunoblot analysis and quantitative RT-PCR, respectively. The amounts of mRNA were normalized as ratios to the amount of $18 \mathrm{~S}$ rRNA. Data are represented as mean \pm SD. Vector control, control vector transfectants. ${ }^{*} \mathrm{P}<0.001$, as evaluated by t-test. Effect of CDK1 inhibition on the expression of the phosphorylated survivin protein in the survivin gene transfectant $(C)$. Survivin protein from nuclear extracts was immunoprecipitated at 6 days after transfection by using the anti-survivin antibody. Immunoprecipitates were immunoblotted using the anti-survivin or anti-phosphothreonine antibody. 
A

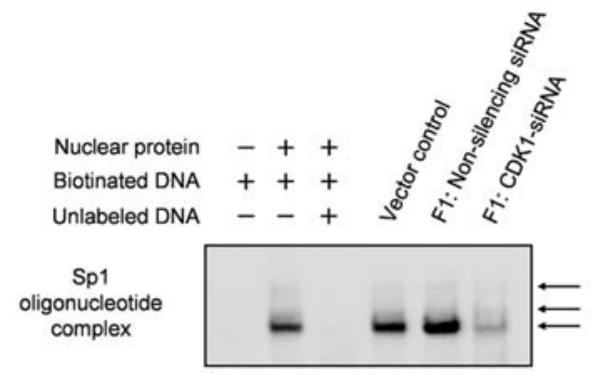

B

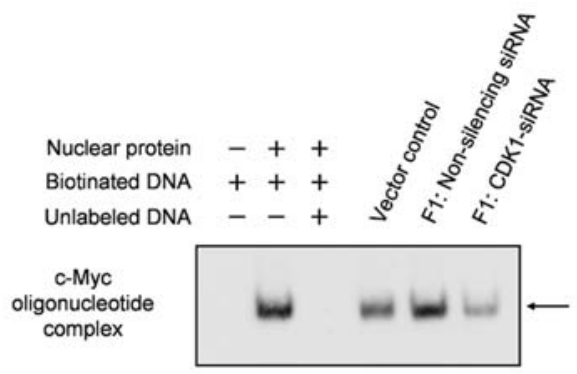

Figure 6. Enhanced DNA binding of Sp1 and c-Myc to the hTERT gene promoter in the survivin gene transfectant was decreased by the suppression of survivin phosphorylation. The survivin gene transfectant F1 was transfected with non-silencing siRNA, which does not share its homology with any known mammalian gene, or siRNA targeting the CDK1 gene (CDK1-siRNA). DNA binding of Sp1 (A) and c-Myc (B) to the hTERT gene promoter was examined at 6 days after transfection by EMSA. Arrows indicate the protein-oligonucleotide complexes. Vector control, control vector transfectants.

was analyzed by immunoblot analysis using the antiphosphothreonine antibody. Although we could detect only the phosphorylated endogenous survivin protein, we observed that this phosphorylation was attenuated in parallel with a decrease in the level of CDK1 protein expression. These results indicate that phosphorylation of the survivin protein by CDK1 is an important post-transcriptional modification for the up-regulation of hTERT expression by survivin.

Survivin phosphorylation was required for the promotion of Spl-and c-Myc-mediated hTERT gene transcription by survivin. Reduction of hTERT mRNA expression by the introduction of CDK1-siRNA indicates that the phosphorylation of the survivin protein may be required for the transcriptional regulation by survivin. To investigate this possibility, we examined the DNA binding of Sp1 and c-Myc to the hTERT gene promoter in the CDK1-siRNA transfectants by using EMSA (Fig. 6A and B). Enhanced binding observed in the survivin gene transfectants was markedly attenuated by the introduction of CDK1-siRNA transfection. We then determined the effect of CDK1-siRNA on the phosphorylation of Sp1 and the c-Myc protein (Fig. 7A and B). Expression of phosphorylated $\mathrm{Sp} 1$ and c-myc protein was decreased in the CDK1-siRNA transfectant as compared to the non-silencing siRNA transfectant.

Interaction between survivin and aurora-B kinase is abrogated by the inhibition of survivin phosphorylation. ABK-siRNA and CDK1-siRNA showed similar effects on the survivininduced up-regulation of hTERT expression. Therefore, we
$\mathbf{A}$

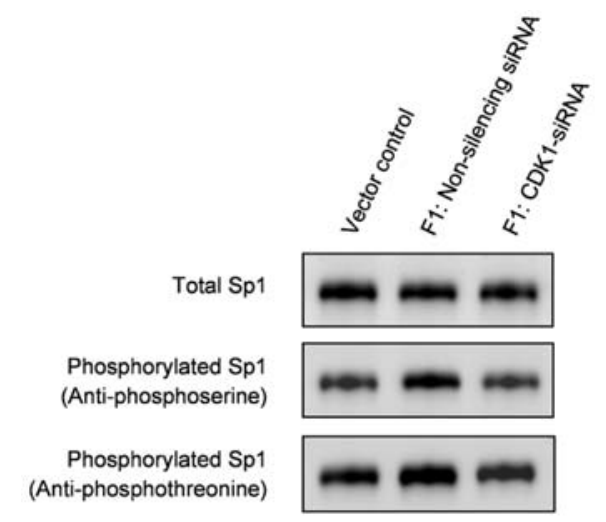

B

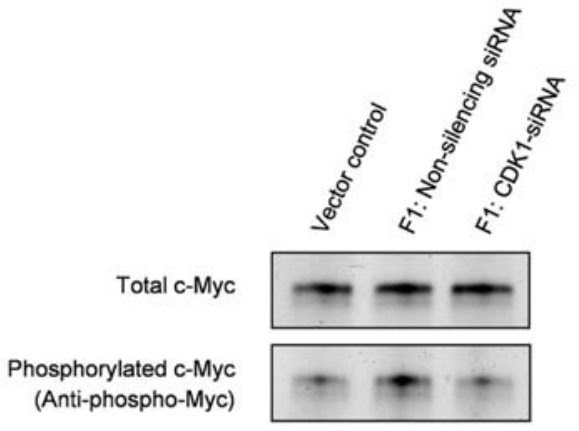

Figure 7. Survivin-mediated phosphorylation of Sp1 and c-Myc protein was attenuated by suppression of survivin phosphorylation. The survivin gene transfectant F1 was transfected with non-silencing siRNA, which does not share its homology with any known mammalian gene, or siRNA targeting the CDK1 gene (CDK1-siRNA). Sp1 protein (A) and c-Myc protein (B) from the nuclear extracts were immunoprecipitated at 6 days after transfection by using the anti-Sp1 or anti-c-Myc antibody, respectively. Sp1 immunoprecipitates and c-Myc immunoprecipitates were immunoblotted using the anti-Sp1, anti-phosphoserine, or anti-phosphothreonine antibody, and anti-c-Myc or anti-phosphorylated c-Myc antibody, respectively. Vector control, control vector transfectants.

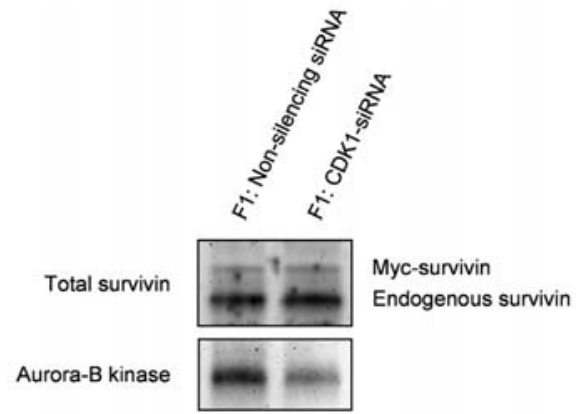

Figure 8. Suppression of survivin phosphorylation abrogated the interaction between survivin and aurora-B kinase. The survivin gene transfectant F1 was transfected with non-silencing siRNA, which does not share its homology with any known mammalian gene, or siRNA targeting the CDK1 gene (CDK1-siRNA). Survivin protein from the nuclear extracts was immunoprecipitated at 6 days after transfection by using the anti-survivin antibody. Immunoprecipitates were immunoblotted using the anti-survivin or anti-aurora-B kinase antibody.

hypothesized that there is an inter-relationship between aurora-B kinase function and survivin phosphorylation. Since the activation of aurora-B kinase is induced by interaction with survivin $(17,18)$, we next determined the binding of aurora-B 
kinase to survivin in CDK1-siRNA transfectants. Survivin proteins were immunoprecipitated from the nuclear extracts of transfectants by using an anti-survivin antibody. After adjusting the samples to ensure that they contained an equal amount of survivin protein, aurora-B kinase protein expression was analyzed by immunoblot analysis using the anti-aurora-B kinase antibody. As shown in Fig. 8, the amount of aurora-B kinase protein that bound to survivin was decreased in the CDK1-siRNA transfectant as compared to the non-silencing siRNA transfectant. This observation suggests that survivin phosphorylation may be required for the interaction between survivin and aurora-B kinase.

\section{Discussion}

In this study, we showed that aurora-B kinase plays an important role in the survivin-mediated up-regulation of hTERT expression. Since inhibition of the aurora-B kinase protein resulted in the suppression of $\mathrm{Sp} 1$ and $\mathrm{c}-\mathrm{Myc}$ phosphorylation, aurora-B kinase may regulate their phosphorylation. However, whether aurora-B kinase directly phosphorylates these two transcriptional factors remains unclear. It has been reported that aurora-B kinase phosphorylates several proteins at serine residues and these proteins include histone $\mathrm{H} 3$, MCAK, Incenp, MgCRacGAP, CENP-A and MKLP1 (27-31). However, all these nuclear proteins are involved in mitotic regulation and our literature search did not identify any study stating that aurora-B kinase phosphorylates transcriptional factors. Recently, Sun et al demonstrated that aurora-A kinase, another member of the aurora kinase family, regulates $\mathrm{NF}-\kappa \mathrm{B}$ via IкB $\alpha$ phosphorylation (32). Although the functions of aurora-A and aurora-B kinase are known to be divergent, the central catalytic domains of these two proteins share substantial homology (33); therefore, it is plausible to consider that aurora-B kinase is involved in the phosphorylation of $\mathrm{Sp} 1$ and c-Myc.

Interestingly, Yang et al demonstrated that overexpression of aurora-A kinase in ovarian and breast epithelial cells induced up-regulation of hTERT expression via enhancing the hTERT gene promoter activity (34). In addition, they observed elevated expression of the c-Myc protein in aurora-A kinase overexpressed cells. Since the level of c-Myc protein expression was not influenced by the introduction of aurora-B kinase siRNA in this study (data not shown), we believe that aurora-B kinase might not be involved in the up-regulation of c-Myc expression. Considering these results, we may conclude that aurora kinases appear to function not only as mitotic but also as transcriptional regulator of many genes.

We also demonstrated that phosphorylation of survivin at the threonine residue by CDK1 is an important step in the interaction between survivin and aurora-B kinase. When we determined the expression of phosphorylated survivin in survivin gene transfectants, we were able to detect only the phosphorylated endogenous survivin protein. The reason for this observation is unclear. Since the anti-survivin antibody used for immunoblot analysis in this study was not available for immunoprecipitation, we used another kind of anti-survivin antibody for immunoprecipitation. As shown in Fig. 1B, immunoblot analysis showed that the expression of the myctagged survivin protein was predominant over the endogenous survivin protein in the survivin gene transfectant F1. In contrast, immunoprecipitation immunoblot analysis showed that expression of the endogenous survivin protein was predominant over the myc-tagged survivin protein (Fig. 5C). Therefore, there is a possibility that myc-tag may interfere with the binding of the anti-survivin antibody that was used in immunoprecipitation.

O'connor et al demonstrated that phosphorylation of survivin at threonine 34 by CDK1 was required for the inhibition of apoptosis during cell division (26). Since phosphorylated survivin binds to caspase- 9 at midbodies during cytokinesis and mutant survivin with a substitution at threonine 34 to alanine, failed to interact with caspase-9, it was suggested that phosphorylation at threonine 34 may increase the affinity of survivin for caspase-9. Therefore, phosphorylation at threonine 34 may increase the affinity of this domain for aurora-B kinase.

Keeping in mind the differential expression of survivin in tumor tissues versus normal tissues and the potential requirement of survivin for maintaining cancer-cell viability, considerable efforts have been made by researchers to use survivin as a target for cancer therapeutics with the dual aim of inhibiting tumor growth and enhancing tumor cell response to apoptosis-inducing agents. Different types of survivin molecular antagonists, including antisense oligonucleotides, ribozymes, siRNAs and dominant-negative mutants, have been used. The introduction of these molecules into cancer cells successfully induced apoptosis and enhanced the effect of anticancer agents and irradiation (35-37). However, these approaches may not be developed for application in routine clinical therapy for cancer until a technique efficiently introducing survivin molecular antagonists into cancer cells is established. Our data indicate that a selective antagonist of aurora-B kinase or CDK1 may attenuate the survivin function without the down-regulation of survivin expression.

\section{References}

1. Tanaka K, Iwamoto S, Gon G, Nohara T, Iwamoto M and Tanigawa N: Expression of survivin and its relationship to loss of apoptosis in breast carcinomas. Clin Cancer Res 6: 127-134, 2000.

2. Kato J, Kuwabara Y, Mitani M, et al: Expression of survivin in esophageal cancer: correlation with the prognosis and response to chemotherapy. Int J Cancer 20: 9592-9595, 2001.

3. Lu CD, Altieri DC and Tanigawa N: Expression of a novel antiapoptosis gene, survivin, correlated with tumor cell apoptosis and p53 accumulation in gastric carcinomas. Cancer Res 58: 1808-1812, 1998.

4. Nakamura M, Tsuji N, Asanuma K, et al: Survivin as a predictor of cis-diammine- dichloroplatinum sensitivity in gastric cancer patients. Cancer Sci 95: 44-51, 2004.

5. Kawasaki H, Altieri DC, Lu CD, Toyoda M, Tenjo T and Tanigawa N: Inhibition of apoptosis by survivin predicts shorter survival rates in colorectal cancer. Cancer Res 58: 5071-5074, 1998.

6. Sarela AI, Macadam RC, Farmery SM, Markham AF and Guillou PJ: Expression of the antiapoptosis gene, survivin, predicts death from recurrent colorectal carcinoma. Gut 46: 645-650, 2006.

7. Satoh K, Kaneko K, Hirota M, Masamune A, Satoh A and Shimosegawa T: Expression of survivin is correlated with cancer cell apoptosis and is involved in the development of human pancreatic duct cell tumors. Cancer 92: 271-278, 2001.

8. Ikeguchi M, Ueta T, Yamane Y, Hirooka Y and Kaibara N: Inducible nitric oxide synthase and survivin messenger RNA expression in hepatocellular carcinoma. Clin Cancer Res 8: 3131-3136, 2002. 
9. Saitoh Y, Yaginuma Y and Ishikawa M: Analysis of Bcl-2, Bax and Survivin genes in uterine cancer. Int J Oncol 15: 137-141, 1999.

10. Yoshida H, Ishiko O, Sumi T, Matsumoto Y and Ogita S: Survivin, bcl-2 and matrix metalloproteinase-2 enhance progression of clear cell- and serous-type ovarian carcinomas. Int J Oncol 19: 537-542, 2004.

11. Adida C, Recher C, Raffoux E, et al: Expression and prognostic significance of survivin in de novo acute myeloid leukaemia. $\mathrm{Br}$ J Haematol 111: 196-203, 2000.

12. Tamm I, Wang Y, Sausville E, et al: IAP-family protein survivin inhibits caspase activity and apoptosis induced by Fas (CD95), Bax, caspases, and anticancer drugs. Cancer Res 58: 5315-5320, 1998.

13. Shin S, Sung BJ, Cho YS, et al: An anti-apoptotic protein human survivin is a direct inhibitor of caspase-3 and -7 . Biochemistry 40: 1117-1123, 2001.

14. Asanuma K, Moriai R, Yajima T, et al: Survivin as a radioresistance factor in pancreatic cancer. Jpn J Cancer Res 91: 1204-1209, 2001.

15. Asanuma K, Kobayashi D, Furuya D, Tsuji N, Yagihashi A and Watanabe N: A role for survivin in radioresistance of pancreatic cancer cells. Jpn J Cancer Res 93: 1057-1062, 2002

16. Li F, Ambrosini G, Chu EY, Plescia J, Tognin S, Marchisio PC and Altieri DC: Control of apoptosis and mitotic spindle checkpoint by surviving. Nature 396: 580-584, 1998.

17. Bolton MA, Lan W, Powers SE, McCleland ML, Kuang J and Stukenberg PT: Aurora B kinase exists in a complex with survivin and INCENP and its kinase activity is stimulated by survivin binding and phosphorylation. Mol Biol Cell 13: 3064-3077, 2002.

18. Chen J, Jin S, Tahir SK, Zhang H, et al: Survivin enhances aurora-B kinase activity and localizes aurora-B in human cells. J Biol Chem 278: 486-490, 2003.

19. Asanuma K, Tsuji N. Endoh T, Yagihashi A and Watanabe N: Survivin enhances Fas ligand expression via up-regulation of Sp1-mediated gene transcription in colon cancer cells. J Immunol 172: 3922-3929, 2004.

20. Endoh T, Tsuji N, Asanuma K, Yagihashi A and Watanabe N: Survivin enhances telomerase activity via up-regulation of specificity protein 1- and c-Myc-mediated human telomerase reverse transcriptase gene transcription. Exp Cell Res 305: 300-311, 2005

21. Mahotka C, Wenzel M, Springer E, Gabbert HE and Gerharz CD: Survivin-deltaEx3 and survivin-2B: two novel splice variants of the apoptosis inhibitor survivin with different antiapoptotic properties. Cancer Res 59: 6097-6102, 1999.

22. Kyo S, Takakura M, Taira T, et al: Sp1 cooperates with c-Myc to activate transcription of the human telomerase reverse transcriptase gene (hTERT). Nucleic Acids Res 28: 669-677, 2000 .
23. Kavurma MM, Santiago FS, Bonfoco E and Khachigian LM: Sp1 phosphorylation regulates apoptosis via extracellular FasLFas engagement. J Biol Chem 276: 4964-4971, 2001.

24. Ye X and Liu SF: Lipopolysaccharide down-regulates Sp1 binding activity by promoting $\mathrm{Sp} 1$ protein dephosphorylation and degradation. J Biol Chem 277: 31863-31870, 2002.

25. Gupta S, Seth A and Davis RJ: Transactivation of gene expression by Myc is inhibited by mutation at the phosphorylation sites Thr-58 and Ser-62. Proc Natl Acad Sci USA 90: 3216-3220, 1993.

26. O'Connor DS, Grossman D, Plescia J, et al: Regulation of apoptosis at cell division by $\mathrm{p} 34 \mathrm{cdc} 2$ phosphorylation of survivin. Proc Natl Acad Sci USA 97: 13103-13107, 2000.

27. Goto H, Yasui Y, Nigg EA and Inagaki M: Aurora-B phosphorylates Histone $\mathrm{H} 3$ at serine 28 with regard to the mitotic chromosome condensation. Genes Cells 7: 11-17, 2002.

28. Ohi R, Sapra T, Howard J and Mitchison TJ: Differentiation of cytoplasmic and meiotic spindle assembly MCAK functions by Aurora B-dependent phosphorylation. Mol Biol Cell 15: 2895-2906, 2004.

29. Bishop JD and Schumacher JM: Phosphorylation of the carboxyl terminus of inner centromere protein (INCENP) by the Aurora B Kinase stimulates Aurora B kinase activity. J Biol Chem 277: 27577-27580, 2002.

30. Ban R, Irino Y, Fukami $\mathrm{K}$ and Tanaka $\mathrm{H}$ : Human mitotic spindle-associated protein PRC1 inhibits MgcRacGAP activity toward Cdc42 during the metaphase. J Biol Chem 279: 16394-16402, 2004.

31. Zeitlin SG, Shelby RD and Sullivan KF: CENP-A is phosphorylated by Aurora B kinase and plays an unexpected role in completion of cytokinesis. J Cell Biol 155: 1147-1157, 2001.

32. Sun C, Chan F, Briassouli P and Linardopoulos S: Aurora kinase inhibition down- regulates NF-kappaB and sensitises tumour cells to chemotherapeutic agents. Biochem Biophys Res Commun 352: 220-225, 2007

33. Scrittori L, Skoufias DA, Hans F, et al: A small C-terminal sequence of Aurora B is responsible for localization and function. Mol Biol Cell 16: 292-305, 2005.

34. Yang H, Ou CC, Feldman RI, Nicosia SV, Kruk PA and Cheng JQ: Aurora-A kinase regulates telomerase activity through c-Myc in human ovarian and breast epithelial cells. Cancer Res 64: 463-467, 2004.

35. Altieri DC: Validating survivin as a cancer therapeutic target. Nat Rev Cancer 3: 46-54, 2003.

36. Li F: Survivin study: what is the next wave? J Cell Physiol 197: 8-29, 2003.

37. Zaffaroni N, Pennati M and Daidone MG: Survivin as a target for new anticancer interventions. J Cell Mol Med 9: 360-372, 2005. 